\title{
Naive Pregerminal Center B-Lymphocyte
}

National Cancer Institute

\section{Source}

National Cancer Institute. Naive Pregerminal Center B-Lymphocyte. NCI Thesaurus.

Code C38337.

A mature $\mathrm{B}$ lymphocyte that expresses $\lg \mathrm{M}$ and $\lg \mathrm{D}$. It is found in peripheral blood, lymph nodes and secondary lymphoid organs, but has not moved into the germinal center of lymphoid organs. Once this cell encounters an antigen in the presence of helper T cells and becomes activated, it moves to the next stage of B-lymphocyte development. 